\title{
Ferroelectricity and tunneling electroresistance effect in asymmetric ferroelectric tunnel junctions
}

\author{
L. L. Tao and J. Wang ${ }^{\text {a) }}$ \\ Department of Physics and the Center of Theoretical and Computational Physics, \\ The University of Hong Kong, Hong Kong, China
}

(Received 31 March 2016; accepted 28 May 2016; published online 13 June 2016)

\begin{abstract}
We report the investigation on the ferroelectricity and tunneling electroresistance (TER) effect in $\mathrm{PbTiO}_{3}$ (PTO)-based ferroelectric tunnel junctions (FTJs) using first-principles calculations. For symmetric FTJs, we have calculated the average polarizations of PTO film and effective screening lengths of different metal electrodes for a number of FTJs, which is useful for experimental research. For asymmetric FTJs, significant asymmetric ferroelectric displacements in PTO film are observed, which is attributed to the intrinsic field generated by the two dissimilar electrodes. Moreover, by performing quantum transport calculations on those asymmetric FTJs, a sizable TER effect is observed. It is found that the asymmetry of ferroelectric displacements in PTO barrier, which is determined by the difference of work functions of the electrodes, controls the observed TER effect. Our results will help unravel the TER mechanism of asymmetric FTJs in most experiments and will be useful for the designing of FTJ-based devices. Published by AIP Publishing.
\end{abstract}

[http://dx.doi.org/10.1063/1.4953642]

\section{INTRODUCTION}

The research on the ferroelectric (FE) materials has attracted great interest due to the potential technological applications of FE materials in electronic devices, such as nonvolatile random access memories, logic devices, sensors, etc. ${ }^{1}$ Remarkably, the discovery of nanoscale-thick ferroelectric film ${ }^{2-4}$ has aroused the investigations of ferroelectric tunnel junctions (FTJs). ${ }^{5,6}$ A typical FTJ is made of two metal electrodes sandwiching a thin ferroelectric film maintaining a macroscopic polarization, which serves to modulate the electron tunneling probability through the barrier. The change in resistance caused by polarization reversal is defined as the well-known tunneling electroresistance (TER) effect. $^{5,6}$ It is generally accepted that the asymmetry of an FTJ in order to obtain a sizable TER can be achieved either using asymmetric electrodes ${ }^{7-9}$ or through barrier interface engineering. ${ }^{10-13}$ Indeed, the giant TER effect has been reported in $\mathrm{BaTiO}_{3}(\mathrm{BTO})^{4,10,12,14}$ or $\mathrm{PbTiO}_{3}(\mathrm{PTO})^{15}$ based asymmetric FTJs by many experimental groups. On the other hand, it is more interesting to construct the multiferroic tunnel junctions (MFTJs), i.e., an FTJ with ferromagnetic electrodes. For an MFTJ, the resistance depends on both the magnetic configurations of the two electrodes and polarization orientation of the FE barrier, and consequently, a fourstate resistance can be achieved in an MFTJ element, as has been reported by experimental studies. ${ }^{16,17}$

In most experiments, the asymmetric FTJs (a-FTJs in the following) are fabricated using asymmetric electrodes, namely, the bottom electrodes are commonly conductive oxides such as $\mathrm{SrRuO}_{3}$ (SRO), ${ }^{15} \mathrm{La}_{1-x} \mathrm{Sr}_{x} \mathrm{MnO}_{3}$ (LSMO), ${ }^{4,9,12}$ or $\mathrm{Nb}$ doped $\mathrm{SrTiO}_{3}$ (STO $)^{14}$ grown on a thick STO substrate while the top electrodes are metals such as Pt or Au. ${ }^{9}$ Thus it is

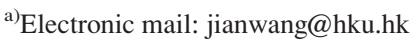

inevitable to induce an intrinsic built-in electric field $E_{b i}$ through FE films due to the difference of work functions (WFs) of electrodes. ${ }^{18}$ The free energy contributed by the $E_{b i}$ is of the form $-\overrightarrow{E_{b i}} \cdot \vec{P}$, suggesting that it is energetically favorable while the polarization $\vec{P}$ is parallel to $\overrightarrow{E_{b i}}$ and consequently an asymmetric double-well potential is expected. ${ }^{19}$ As such $E_{b i}$ can enhance or suppress the average polarization magnitude, which manifests itself in asymmetric displacements in FE film for two different polarization states. This phenomenon was confirmed in the model systems of SRO/PTO/ $/ \mathrm{Pt}^{20}$ and $\mathrm{Fe} /$ $\mathrm{PTO} / \mathrm{Pt}^{21}$ based on first-principles calculations. If that is indeed the case, a sizable TER effect is expected in such a-FTJs and should be explored from parameter-free first-principles simulations. On the other hand, $E_{b i}$ can decrease or even eliminate the depolarization field $E_{d}$ for a given polarization orientation, thus resulting in the vanishing critical thickness for ferroelectricity in the a-FTJs, as confirmed by first-principles calculations. ${ }^{22}$ Luo et al. ${ }^{23}$ investigated a representative a-FTJ model, that is, SRO/BTO/Pt based on quantum transport calculations, and found that the effective potential barrier can be modified by the polarization, thus giving rise to the giant TER effect. A similar TER effect was also demonstrated in LSMO/BTO/Au and LSMO/BTO/Cu a-FTJs experimentally. ${ }^{9}$ Despite these advances, to date, there are very limited theoretical works about understanding the ferroelectricity and especially the TER mechanism in such a-FTJs, which motivates our present work.

In this work, we systematically studied the ferroelectricity for a number of PTO-based FTJs, including both symmetric FTJs (s-FTJs) with identical left and right electrodes and a-FTJs with different left and right electrodes by means of first-principles calculations. Specifically, Au, Pt, Fe, Co, and SRO electrodes were used to investigate the ferroelectricity of s-FTJs or a-FTJs. We calculated the metal-oxygen relative displacements for s-FTJs which are directly related to the 
polarizations and evaluated screening lengths of different metals by calculating the depolarizing field within the PTO film. In the case of a-FTJs, that is, $\mathrm{Pt} / \mathrm{PTO} / \mathrm{Fe}$ or $\mathrm{SRO} / \mathrm{PTO} /$ $\mathrm{Pt}$, we found significant asymmetric displacements within PTO film caused by the intrinsic field, which is in line with previous results. ${ }^{20,21}$ Using the $\mathrm{Pt} / \mathrm{PTO} / \mathrm{Fe}$ and $\mathrm{SRO} / \mathrm{PTO} / \mathrm{Pt}$ FTJs as prototypical examples, we predicted an order of magnitude difference in conductance between two different polarization states by performing the quantum transport calculations. The microscopic physics of TER mechanism was identified and understood through the analysis of the realspace scattering states and complex band structure.

\section{COMPUTATIONAL METHOD AND DETAILS}

Our atomic and electronic structures calculations were performed using the projector-augmented wave (PAW) method ${ }^{24}$ as implemented in the Vienna ab-initio simulation package (VASP). ${ }^{25}$ An energy cutoff of $500 \mathrm{eV}$ and local density approximation (LDA) ${ }^{26}$ for the exchange and correlation functional were used throughout. Note that Hubbard-U correction $(\mathrm{LDA}+\mathrm{U})$ for the transition element $d$ orbital such as Ti-3d orbital was not taken into account in our calculations, because it was shown that the LDA $+\mathrm{U}$ method yields significantly worse structural parameters for PTO than the LDA method in comparison with the experimental results. ${ }^{27}$ The calculated lattice constants are $a=3.866 \AA$ and $c=4.038 \AA$ for PTO, in good agreement with previous results. ${ }^{28}$ The calculated polarization is $83.16 \mu \mathrm{C} / \mathrm{cm}^{2}$. The supercell model for s-FTJs studied in this work consists of 6.5 unit cells of PTO and 10.5 unit cells for Fe and Co electrodes, 9.5 unit cells for Pt and Au electrodes, and 6.5 unit cells of SRO due to their different out-of-plane lattice constants as listed in Table I. Note that the thicknesses of electrodes should be sufficiently large to ensure that: (i) the coupling between the left and right electrode/FE interfaces is negligible; (ii) the structural parameters of the interior of the electrode region are close to that of bulk; (iii) the macroscopic electrostatic potential profile in the interior of the electrode region is almost flat to ensure that the electric field is completely screened. The in-plane lattice constant $a$ of the junction is fixed at $3.866 \AA$. Atomic relaxations were performed using a $6 \times 6 \times 1 k$-point mesh until the forces on each atom were less than $0.01 \mathrm{eV} / \mathrm{A}$. To investigate two possible terminated interfaces of $\mathrm{PTO}(001)$, both $\mathrm{TiO}$ and $\mathrm{PbO}$ terminations [see Fig. 1] were considered for the interface structures.

TABLE I. Fully relaxed bulk tetragonality $c / a(a=3.866 \AA)$ for a number of metal electrodes. Calculated average polarization $\bar{P}$ of PTO film and effective screening lengths $\lambda_{\text {eff }}$ of metal electrodes for the corresponding symmetric FTJs with either $\mathrm{TiO}$ or $\mathrm{PbO}$ termination.

\begin{tabular}{lcccccc}
\hline \hline & & \multicolumn{2}{c}{$\bar{P}\left(\mu \mathrm{C} / \mathrm{cm}^{2}\right)$} & & \multicolumn{2}{c}{$\lambda_{\text {eff }}(\AA)$} \\
\cline { 7 - 8 } \cline { 7 - 7 } Electrode & $c / a$ & TiO term & PbO term & & TiO term & PbO term \\
\hline $\mathrm{Au}$ & 1.138 & 65.76 & 64.09 & & 0.071 & 0.063 \\
$\mathrm{Pt}$ & 1.025 & 71.99 & 80.15 & & 0.062 & 0.013 \\
$\mathrm{Fe}$ & 1.010 & 71.01 & 90.52 & & 0.064 & 0.029 \\
$\mathrm{Co}$ & 0.998 & 64.37 & 85.98 & & 0.070 & 0.034 \\
$\mathrm{SRO}$ & 1.003 & 35.82 & 56.43 & & 0.125 & 0.081 \\
\hline \hline
\end{tabular}

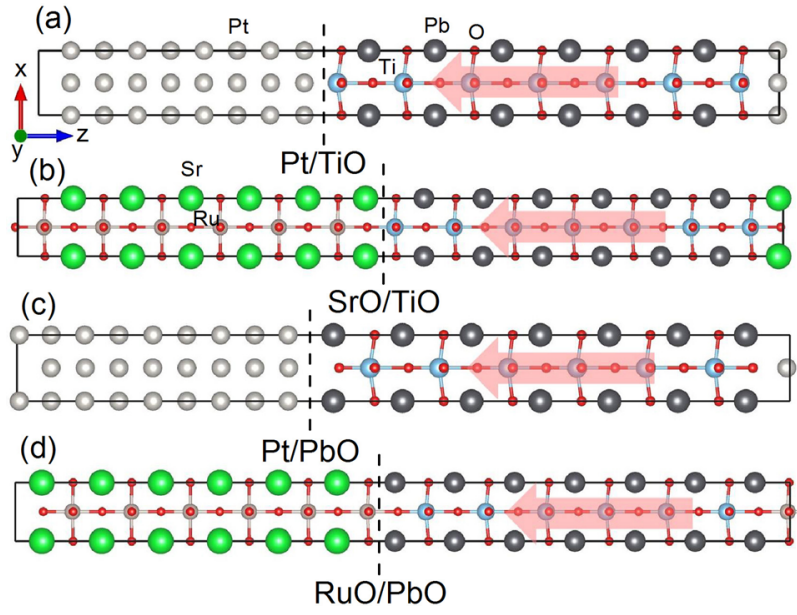

FIG. 1. Fully relaxed atomic structure of s-FTJs for left polarization state as highlighted by the red arrows. (a, c) Pt/PTO/Pt junction. (b, d) SRO/PTO/ SRO junction. Two interface terminations studied: (a, b) TiO-termination type and (c, d) PbO-termination type. The interface positions are marked by the dashed lines.

Our quantum transport calculations are based on the state-of-the-art technique nonequilibrium Green's function (NEGF)-density functional theory (DFT) by combining real-space density functional theory (DFT) with the Keldysh nonequilibrium Green's function (NEGF) formalism, as implemented in Nanodcal package. ${ }^{29}$ The zero-bias conductance $G$ is given by Landauer-Büttiker formula

$$
G=\frac{2 e^{2}}{h} \sum_{\mathbf{k}_{\|}} T\left(\mathbf{k}_{\|}, E_{F}\right),
$$

where $T\left(\mathbf{k}_{\|}, E_{F}\right)$ is the transmission coefficient of an electron at the Fermi energy $E_{F}$ with a transverse Bloch wave vector $\mathbf{k}_{\|}=\left(k_{x}, k_{y}\right), e$ the electron charge, and $h$ the Planck's constant. A $10 \times 10 \mathbf{k}_{\|}$mesh for self-consistent calculations and a $100 \times 100 \mathbf{k}_{\|}$mesh for evaluating conductance were employed. The $\mathrm{LDA}^{26}$ for the exchange and correlation potential was used throughout.

\section{RESULTS AND DISCUSSION}

First we investigate the ferroelectricity for s-FTJs whose atomic structures are shown in Fig. 1. Fig. 2 shows the relative metal-O (M-O) displacements within the PTO film for both interface terminations. As can be seen, the displacements are uniform throughout the PTO layer in both $\mathrm{TiO}$ and $\mathrm{PbO}$ terminations giving rise to a ferroelectric state as expected. In addition, the $\mathrm{Pb}-\mathrm{O}$ displacements are larger than Ti-O displacements, as clearly seen from the zigzag lines. More importantly, the M-O displacements strongly depend on the type of metal electrodes and interface terminations, which is in line with previous calculations; Al-Saidi and Rappe ${ }^{30}$ based on first-principles simulations on $\mathrm{Pt} / \mathrm{PTO} / \mathrm{Pt}$ and $\mathrm{Au} / \mathrm{PTO} / \mathrm{Au}$ examples, demonstrated that the FE properties depend on both electrode type and electrode/FE termination. For example, the M-O displacements for an FTJ with SRO electrodes are smaller than those with other electrodes while an FTJ with $\mathrm{Fe}$ electrodes has the largest M-O 

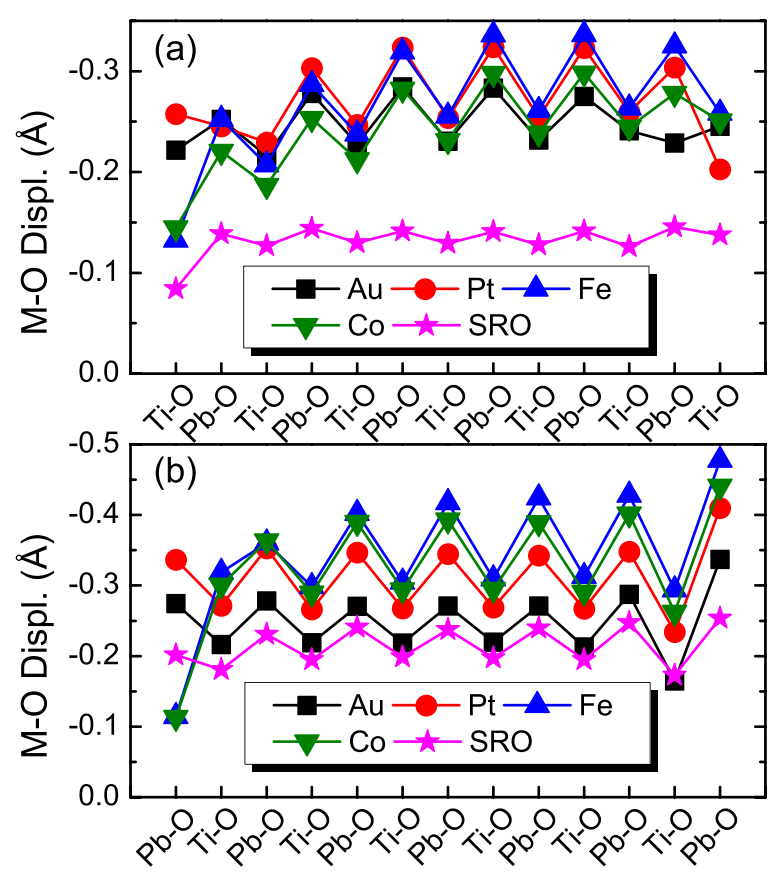

FIG. 2. The relative metal-O (M-O) displacements $\left(z_{M}-z_{O}\right)$ within PTO film with different metal electrodes. (a) $\mathrm{TiO}$ termination and (b) $\mathrm{PbO}$ termination.

displacements in both interface terminations. Additionally, comparing Fig. 2(a) with 2(b), the overall feature is that the displacements are larger for $\mathrm{PbO}$ termination than that for $\mathrm{TiO}$ termination. From those M-O displacements and the Born effective charges, we evaluated the local polarization $P_{k}$ by using ${ }^{31}$

$$
P_{k}=\frac{1}{\Omega_{k}} \sum_{n} Z_{n}^{*} \delta z_{n},
$$

where $P_{k}$ is the polarization of the $k$-th unit cell whose volume is $\Omega_{k}, Z_{n}^{*}$ the Born effective charge of the $n$-th atom, and $\delta z_{n}$ the displacement of the $n$-th atom. The Born effective charges $Z_{n}^{*}$ calculated using density functional perturbation theory $^{32}$ for $\mathrm{Pb}, \mathrm{Ti}, \mathrm{O}_{\perp}$ ( $\mathrm{TiO}$ plane), and $\mathrm{O}_{\|}(\mathrm{PbO}$ plane) ions of tetragonal PTO $(a=3.866 \AA, c=4.038 \AA)$ are, respectively, $3.79,6.69,-2.52$, and -5.43 electrons. For example, the local polarization across PTO film for s-FTJs with Pt and SRO electrodes are shown in Fig. 3. One can see that the magnitude of local polarization is well consistent with that of M-O displacements shown in Fig. 2.

The fact that displacements depend on the type of metal electrode and interface termination is due to the different effective screening lengths $\lambda_{\text {eff }}$ of metals. Using the shortcircuit electrostatic boundary conditions, the depolarizing field $E_{d}$ is given by ${ }^{30,33,34}$

$$
E_{d}=-\frac{2 \lambda_{e f f} \bar{P}}{\epsilon_{0} d}
$$

where $\bar{P}$ is the average polarization obtained by averaging $P_{k}$ in Eq. (2) and $d$ is the PTO film thickness. We evaluated the depolarizing field $E_{d}$ from the slope of the macroscopic electrostatic potential within PTO film. Using Eq. (3), we then

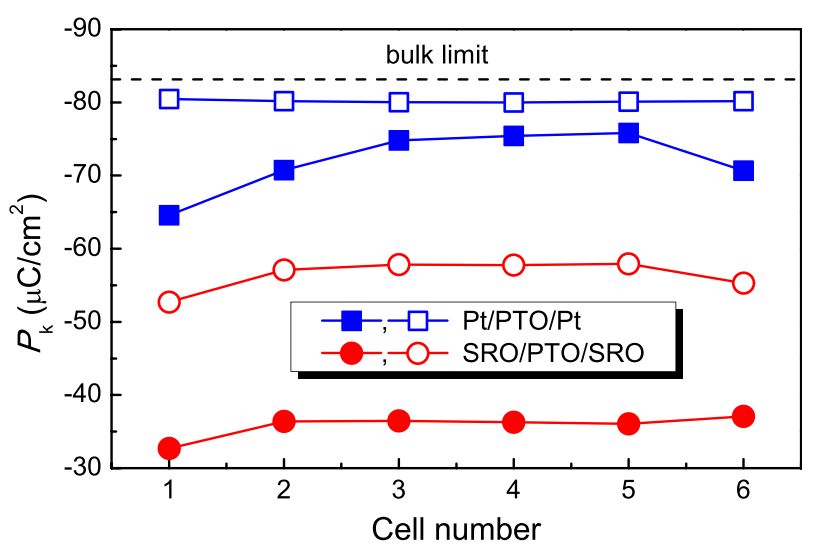

FIG. 3. Local polarization $P_{k}$ mapped onto unit cell evaluated from the Born effective charges. The dashed line denotes the position of bulk polarization for reference. Solid and open symbols stand for TiO termination condition and $\mathrm{PbO}$ termination condition, respectively.

obtained the value of $\lambda_{\text {eff }}$, as summarized in Table I. For example, we found $\lambda_{\text {eff }}$ to be $0.062 \AA$ and $0.125 \AA$ for Pt and $\mathrm{SRO}$ with $\mathrm{TiO}$ termination, and with $\mathrm{PbO}$ termination, $\lambda_{\text {eff }}$ is $0.013 \AA$ for Pt and $0.081 \AA$ for SRO. As a comparison, AlSaidi and Rappe $^{30}$ reported a value of $0.045 \AA$ for $\mathrm{Pt} / \mathrm{PTO} / \mathrm{Pt}$ junction with $\mathrm{TiO}$ termination and $0.007 \AA$ with $\mathrm{PbO}$ termination. Gerra et $a l .{ }^{34}$ reported a value of $0.1 \AA$ for $\mathrm{SRO} /$ $\mathrm{BTO} / \mathrm{SRO}$ junction with TiO termination. It is seen that our results are in good agreement with those reported previously.

We have seen that the FE displacements in PTO film for s-FTJs depend on both metal type and interface termination. To achieve a sizable TER effect, it is instructive to construct a-FTJs with two different electrodes. Without loss of generality, we have constructed two prototypical examples, namely, $\mathrm{Pt} / \mathrm{PTO} / \mathrm{Fe}$ and SRO/PTO/Pt. Note that both interface terminations of a-FTJs are $\mathrm{PbO}$ type, instead of $\mathrm{TiO}$ type in some other calculations. ${ }^{35,36}$ However, the analysis and conclusion in this work are very general and are applicable to TiO termination condition as well. The a-FTJs can be denoted as $\mathrm{Pt} /$ $(\mathrm{PbO}-\mathrm{TiO})_{m} \mathrm{PbO} / \mathrm{Fe}$ and $\mathrm{SRO} /(\mathrm{PbO}-\mathrm{TiO})_{m} \mathrm{PbO} / \mathrm{Pt}$ with $m$ being the number of unit cells for PTO. All the atoms of the aFTJs are fully relaxed using the same method and parameters presented above. We have calculated the FE displacements in the PTO film for $\mathrm{Pt} /(\mathrm{PbO}-\mathrm{TiO})_{m} \mathrm{PbO} / \mathrm{Fe}$ junction with $m=6$ and for $\mathrm{SRO} /(\mathrm{PbO}-\mathrm{TiO})_{m} \mathrm{PbO} / \mathrm{Pt}$ junction with $m=6-9$ as shown in Figs. 4(b) and 4(c), respectively. The foremost result is that the displacement magnitudes are strongly asymmetric in both cases, as evident from the dashed lines which denote the average Ti-O displacements for $m=6$ condition. In the case of $\mathrm{Pt} / \mathrm{PTO} / \mathrm{Fe}$, the Ti-O displacements in the central $\mathrm{TiO}$ layers are about $0.30 \AA$ and $0.24 \AA$ for left and right polarizations, respectively. While in the case of $\mathrm{SRO} / \mathrm{PTO} / \mathrm{Pt}$, the Ti-O displacements are about $0.25 \AA$ and $0.16 \AA$ for left and right polarizations, respectively. Moreover, as shown in Fig. 4(c), the displacements for right polarization states are rather similar for different barrier thickness, as expected from the direction of $E_{b i}$. In contrast, the displacements for left polarization states are enhanced with the increase of $m$, as expected from the reduction of $E_{b i}$. This indicates that the asymmetry of FE displacements is reduced with increasing barrier thickness, which in turn brings an optimal FE barrier thickness for 

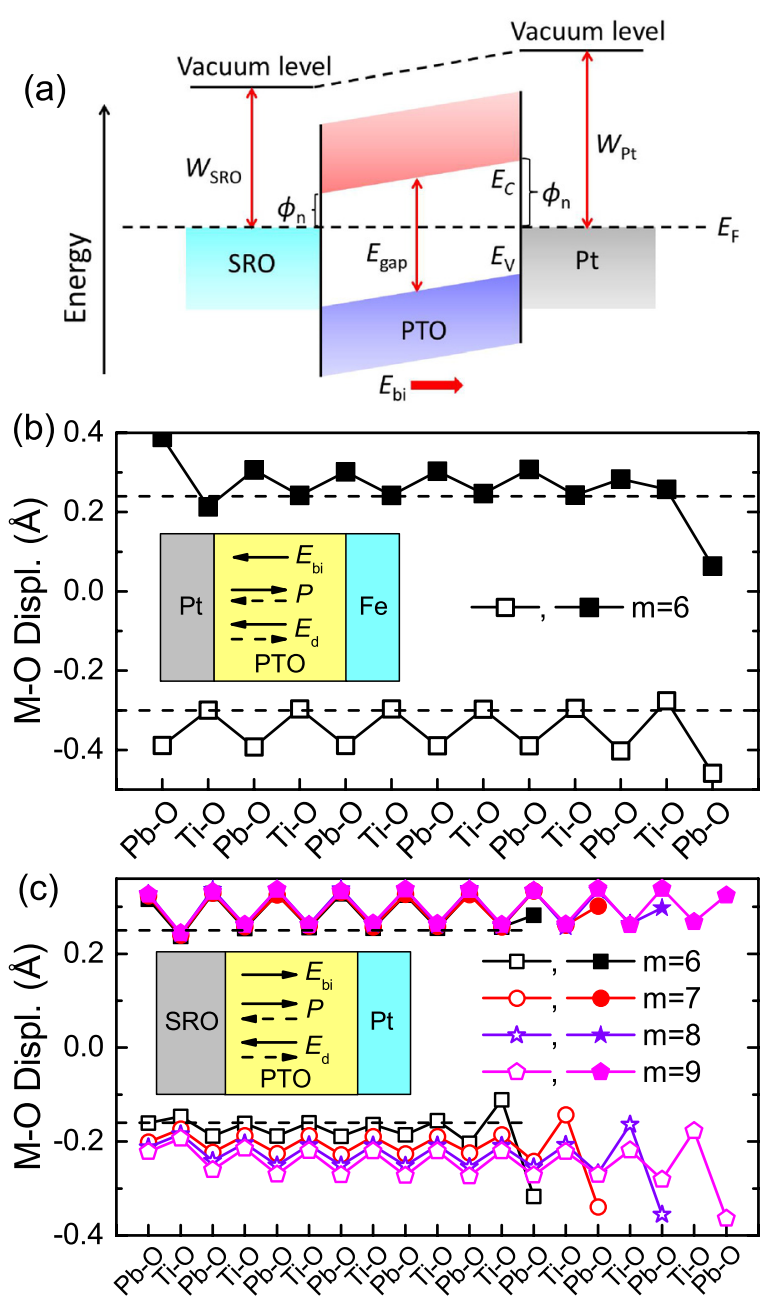

FIG. 4. (a) Schematic band alignment for the SRO/PTO/Pt junction. $W_{S R O}$ and $W_{P t}$ are the work functions of SRO and Pt, respectively. $E_{C}$ and $E_{V}$ are the conduction-band and valence-band edge, respectively. $\phi_{n}$ is the barrier height. $E_{b i}$ is the built-in electric field. (b, c) The relative metal-O (M-O) displacements in the PTO layer for left (open symbols) and right (solid symbols) polarizations. (b) $\mathrm{Pt} / \mathrm{PTO} / \mathrm{Fe}$ junction for $m=6$ and (c) SRO/PTO/Pt junction for $m=6-9$. The dashed lines that denote the average Ti-O displacements for $m=6$ condition are guide to the eyes. Insets: schematic configurations of $E_{b i}$, polarization $P$, and depolarizing field $E_{d}$.

the maximum TER ratio, as will be discussed in the following. Lastly, we find that the displacements are rather homogeneous within the PTO film and yield a reduced polarization as compared with bulk PTO due to the well-known depolarizing field resulting from the incomplete screening by the metal electrodes.

Such asymmetric displacements in a-FTJs are due to the intrinsic built-in electric field $E_{b i}$ produced by dissimilar electrodes having different work functions (WFs) ${ }^{18}$ as mentioned above. Insets of Figs. 4(b) and 4(c) are the schematic configurations of $E_{b i}$, polarization $P$ and depolarizing field $E_{d}$ for a-FTJs. The direction of $E_{b i}$ is pointing from the electrode having lower WF to that having higher WF. ${ }^{18}$ To understand this, taking the SRO/PTO/Pt junction as an example, the band alignment is schematically depicted in Fig. 4(a). When the SRO/PTO/Pt junction is at equilibrium, the Fermi level of the system is flat. In contrast, the vacuum level is not flat due to the work function difference. Thereby, there is a potential drop across the PTO barrier from SRO to Pt. In this work the calculated $\mathrm{WFs}^{37}$ are about $4.41 \mathrm{eV}$, $6.09 \mathrm{eV}$, and $4.85 \mathrm{eV}$ for $\mathrm{Fe}, \mathrm{Pt}$, and $\mathrm{SRO}$, respectively, indicating that $E_{b i}$ is pointing from $\mathrm{Fe}$ to $\mathrm{Pt}$ for $\mathrm{Pt} / \mathrm{PTO} / \mathrm{Fe}$ junction and is pointing from SRO to $\mathrm{Pt}$ for $\mathrm{SRO} / \mathrm{PTO} / \mathrm{Pt}$ junction. To further confirm this, Fig. 5 plots the electrostatic potential profile through the a-FTJ for paraelectric (PE) state (the PE state can eliminate the effect of $E_{d}$ ), that is, an artificial structure without $\mathrm{M}-\mathrm{O}$ relative displacements in PTO with the interface distance determined by the total energy minimization. It can be seen clearly (see the red dashed lines or blue dotted lines) that there is a potential drop throughout the PTO film from Fe to Pt or from SRO to Pt, which is the manifestation of the built-in electric field. We emphasize that the electrostatic model assuming that the built-in field originates from the work function difference of electrodes can be used to qualitatively explain the asymmetric displacements and mono-stable polarization state below a critical thickness observed in the asymmetric FTJs. The details of the electrode/FE interfaces should be fully considered in the actual ferroelectricity calculations and analysis. ${ }^{38}$

We now investigate the TER effect by performing quantum transport calculations. The TER ratio is defined as TER $=\left|G_{\leftarrow}-G_{\rightarrow}\right| / \min \left(G_{\rightarrow}, G_{\leftarrow}\right)$, in which $G_{\leftarrow}\left(G_{\rightarrow}\right)$ is the total conductance for the FTJ in left (right) polarization. We have calculated the conductance and TER ratios for $\mathrm{Pt} /(\mathrm{PbO}-\mathrm{TiO})_{m} \mathrm{PbO} /$ Fe junction with $m=6$ and for $\mathrm{SRO} /(\mathrm{PbO}-\mathrm{TiO})_{m} \mathrm{PbO} / \mathrm{Pt}$ junction with $m=6-9$. We find that $G_{\rightarrow}$ is about one order of magnitude larger than $G_{\leftarrow}$ for $\mathrm{Pt} / \mathrm{PTO} / \mathrm{Fe}$ junction and, as a consequence, a giant TER ratio of more than $800 \%$ is obtained.
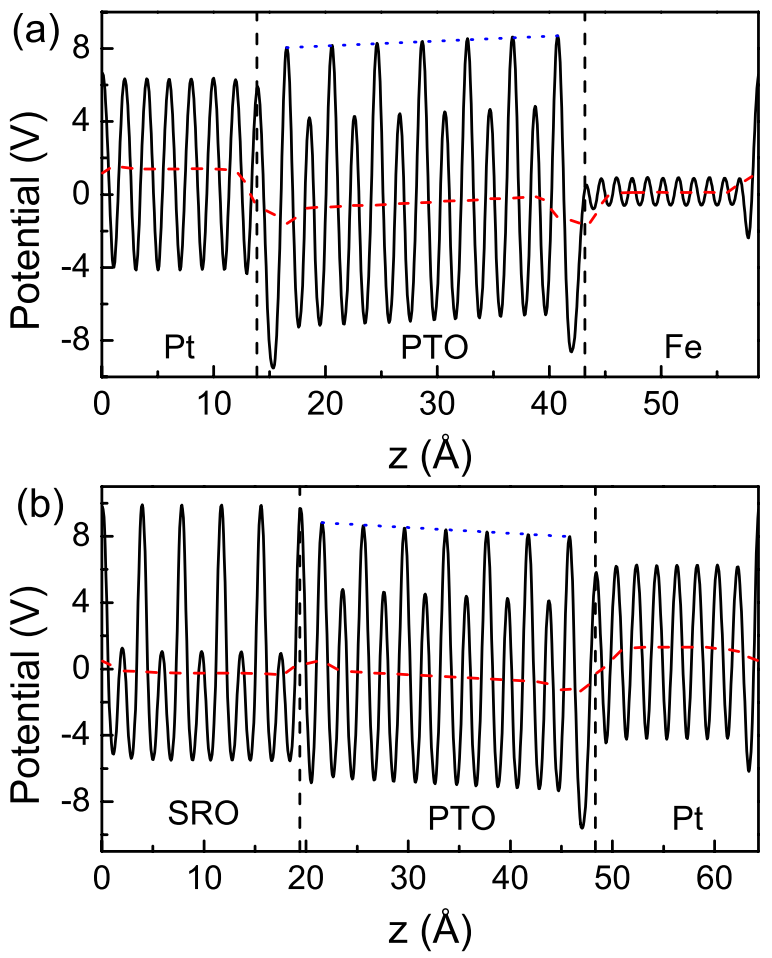

FIG. 5. Planar (black solid lines) and macroscopic averaged (red dashed lines) electrostatic potential along the $z$ direction for the two different aFTJs in paraelectric (PE) state. (a) Pt/PTO/Fe and (b) SRO/PTO/Pt. The vertical dashed lines denote roughly the positions of interfaces. The blue dotted lines that mark the potential drop through PTO film are guide to the eyes. 
Fig. 6 shows the barrier thickness dependent conductance and TER ratios for SRO/PTO/Pt junction with $m=6-9$. The conductance for both left and right polarization states decrease exponentially with increasing barrier thickness, which agrees well with the physics of quantum tunneling. More interestingly, it is found that the TER ratio reaches the largest value at the thickness of $m=7$, as expected from the reduction of the asymmetry of $\mathrm{FE}$ displacements with increasing $m$ [see Fig. 4(c)]. As a comparison, the giant TER effect of as high as $10^{4} \%$ was reported in the asymmetric $\mathrm{La}_{0.67} \mathrm{Sr}_{0.33} \mathrm{MnO}_{3} / \mathrm{BTO} / \mathrm{Co}$ junctions experimentally, ${ }^{39}$ which is one order higher than that of about $10^{3} \%$ in SRO/ PTO/Pt junction in our work. Besides the different systems investigated, there are possibly several uncontrollable parameters in experiment, such as atomic deficiencies, atomic oxidation, lattice misfit and interface quality, etc., which all play important roles in the structure asymmetry and possibly enhance the TER effect. Another example is that a giant TER effect of 5000\% was theoretically predicted in the symmetric SRO/BTO/SRO junction due to significant barrier potential change induced by polarization switching. However, the calculations were based on a phenomenological quantum tunneling model without consideration the realistic atomic details. ${ }^{40}$

To understand the above giant TER effect, it is helpful to find out the factors that account for the change of conductance caused by polarization reversal. When a barrier is not too thin, the transmission coefficient can be approximated as ${ }^{10,35}$

$$
T\left(k_{\|}\right) \approx t_{L}\left(k_{\|}\right) \exp \left[-2 \kappa\left(k_{\|}\right) d\right] t_{R}\left(k_{\|}\right),
$$

where $t_{L}\left(k_{\|}\right)$and $t_{R}\left(k_{\|}\right)$are the interface transmission coefficients characterizing the electron transmission probabilities from electrodes into a barrier across the interface, $\kappa\left(k_{\|}\right)$is the lowest decay rate for an electron with a transverse Bloch wave vector $k_{\|}$, and $d$ is the barrier thickness. According to Eq. (4), it is helpful to follow the change of scattering state through the junction to find out the dominant factor. Scattering states $\psi_{s}$ are eigen-states of the open two-probe device structure linking $z=-\infty$ to $z=+\infty,{ }^{29}$ which is quite intuitive to analyze transport properties. Scattering states $\psi_{s}$, for example, going from left to right can be expressed as

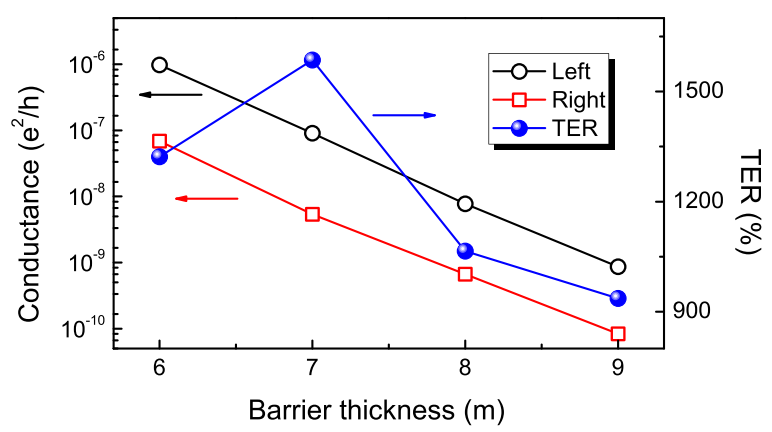

FIG. 6. Barrier thickness dependency of conductance (left axis, in a semilog scale) and TER ratios (right axis) for $\mathrm{SRO} /(\mathrm{PbO}-\mathrm{TiO})_{m} \mathrm{PbO} / \mathrm{Pt}$ junction with $m=6-9$.

$$
\psi_{s}^{n}= \begin{cases}\phi_{L}^{n}+\sum_{m} \varphi_{L}^{m} r^{m n} & \text { inside left electrode }(\mathrm{L}), \\ \psi_{C}^{n} & \text { inside central region }(\mathrm{C}), \\ \sum_{m} \varphi_{R}^{m} t^{m n} & \text { inside right electrode }(\mathrm{R}),\end{cases}
$$

where $r$ and $t$ are the reflection and transmission amplitudes, respectively. The superscript $m$ or $n$ labels the asymptotic Bloch wave vector $k^{m}$ or $k^{n}$ in the electrodes and $\phi, \varphi$ are incoming and outgoing states, respectively. Based on the converged open device Hamiltonian one can obtain reflection and transmission amplitudes and then also the scattering states $\psi_{s}$ by matrix techniques. Taking the SRO/PTO/Pt junction with $m=6$ as an example, Fig. 7(a) plots the decay of the modulus of scattering states $\left|\psi_{s}(z)\right|$ at $\mathbf{k}_{\|}=(0,0)$ through the junction for different polarization orientations. Overall feature seen is that $\left|\psi_{s}(z)\right|$ decays exponentially through the barrier and decays much faster for a-FTJs in right polarization state in comparison with that of left

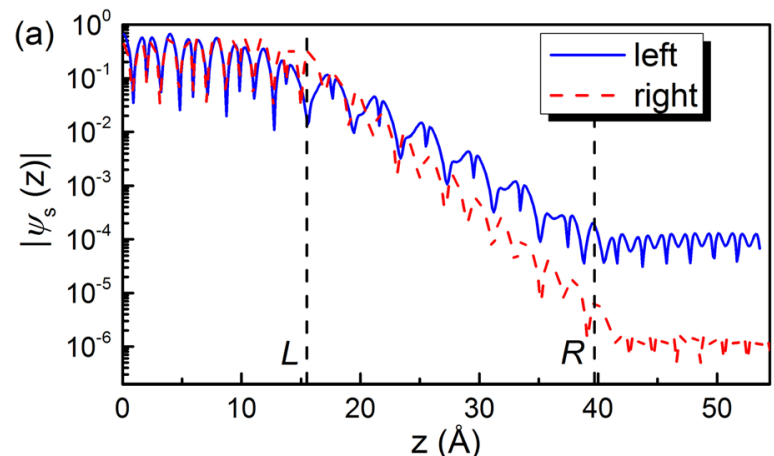

(b)
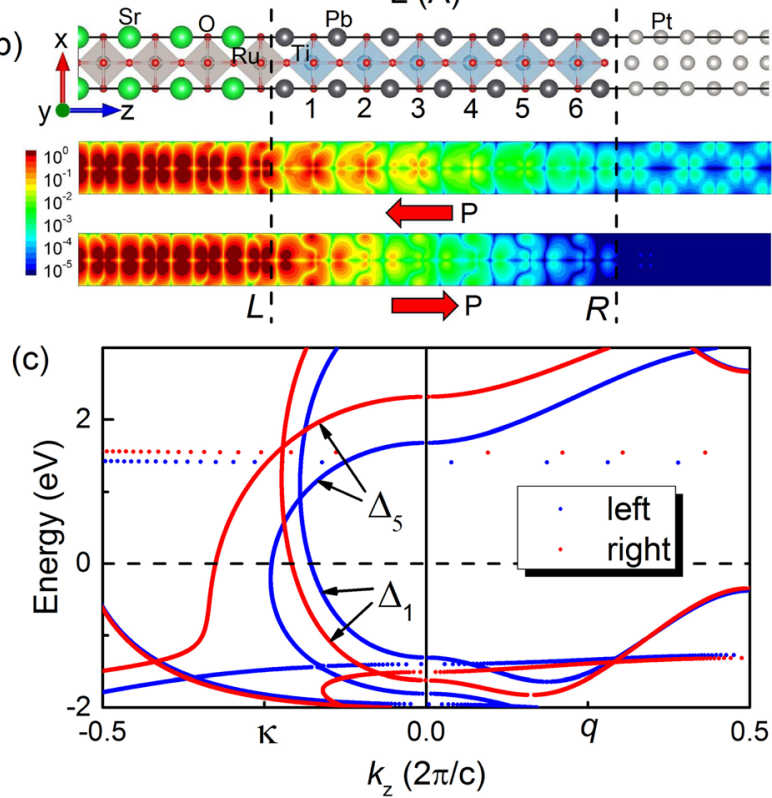

FIG. 7. (a) Distribution of the modulus of scattering states $\left|\psi_{s}(z)\right|$ at $\mathbf{k}_{\|}=(0,0)$ point through the junction for left (black solid lines) and right (red dashed lines) polarization states. The two vertical dashed lines denote roughly the positions of left $(L)$ and right $(R)$ interfaces. (b) Atomic structure of the $\mathrm{SRO} / \mathrm{PTO} / \mathrm{Pt}$ junction and real-space distribution of $\left|\psi_{s}(z)\right|$ through the junction for left and right polarization states. The side bar represents the color coding for the $\left|\psi_{s}(z)\right|$. (c) Complex band structure of bulk PTO for displacement magnitudes corresponding to the left and right polarization states with $m=6$ in Fig. 4(c). The horizontal axes labeled by $q$ (right panel) and $\kappa$ (left panel) correspond to the real and imaginary components of wave vector $k_{z} \equiv q+i \kappa$, respectively. The horizontal dashed line shows the position of the Fermi energy of bulk PTO. 
polarization case. Apparently, it reveals a factor of almost $10^{2}$ difference in the magnitude of $\left|\psi_{s}(z)\right|$ after tunneling through the barrier, which is well consistent with the conductance difference [see Fig. 6]. Further, Fig. 7(b) plots the real-space distribution of $\psi_{s}(z)$ across the junction. We find that $\mathrm{Ti}(\mathrm{Ru})-d$ orbital and O- $p$ orbital $\left(\Delta_{5}\right.$ symmetry states) bond efficiently and form the effective transmission channels. The similar electron transmission channels are also observed for $\mathrm{Pt} / \mathrm{BTO} / \mathrm{Pt}$ junctions. ${ }^{36}$ In addition, $\left|\psi_{s}(z)\right|$ is significantly suppressed within PTO upon turning to right polarization state. On the other hand, as can be seen from the region around interfaces in Figs. 7(a) and 7(b), $t_{L}$ is larger for right polarization than that for left polarization; however, it looks the other way around for $t_{R}$. Therefore the change of $t_{L} t_{R}$ is not crucial and the factor $\exp [-2 \kappa d]$ plays a key role for the giant TER effect. It is worthwhile to note that the factor $\exp [-2 \kappa d]$ dominating the TER effect was also found in the SRO/BTO/SRO junction. ${ }^{10}$

We now investigate the change of decay rate $\kappa$ from the viewpoint of complex band structure. It is known that the Bloch states incoming from the electrode will decay as evanescent states through the barrier in the absence of the interface resonant states. ${ }^{41,42}$ Thus the decay rates of evanescent states determine the tunneling conductance when a barrier is not too thin. The nature of evanescent states can be described by the complex band structure, which extends the usual band structure to include complex Bloch vectors. ${ }^{43,44}$ Fig. 7(c) shows the complex band structure of bulk PTO for displacement magnitudes corresponding to left or right polarization state [see Fig. 4(c) with $m=6$ ] calculated using the Quantum Espresso package. ${ }^{45} \kappa$ is the imaginary component of wave vector $k_{z}$ and determines the decay rates of evanescent states. Namely, the wave functions decay as $\sim e^{-\kappa z}$ through the barrier and the decay length is given by $1 / \kappa$. Clearly, the decay rates (absolute value of $\kappa$ ), in particular, $\Delta_{5}$ states, are enhanced significantly upon the polarization reversal from left to right, which in turn results in the significant transmission reduction, which is in line with the significant suppression of $\left|\psi_{s}(z)\right|$ in right polarization case.

We emphasize that the band gap of $1.4 \mathrm{eV}$ for bulk PTO calculated within DFT-LDA level is smaller than the experimental value of $3.4 \mathrm{eV}$ due to the well-known deficiency of DFT-LDA. As pointed out already, ${ }^{46}$ such underestimation of band gap possibly leads to the charge transfer from metal into the conduction band of the insulator, thus resulting in pathological band alignment and metal ferroelectric states. We checked the Fermi level alignment for SRO/PTO/Pt junction by investigating the layer-resolved projected density of states (PDOS) for Ti- $d$ (forming conduction band) and O$p$ (forming valance band) orbitals, as shown in Fig. 8. We see that the Fermi level lies well inside the reduced band gap for both polarization states, indicating that the interior of PTO film is insulating. The correction of the band gap for PTO will change the calculated conductance quantitatively, but it will not alter our main results qualitatively. We hope to clarify the issue of the underestimation of band gap in a future study.

We should also mention that the effect of the spinorbit coupling (SOC) on the ferroelectricity and TER for
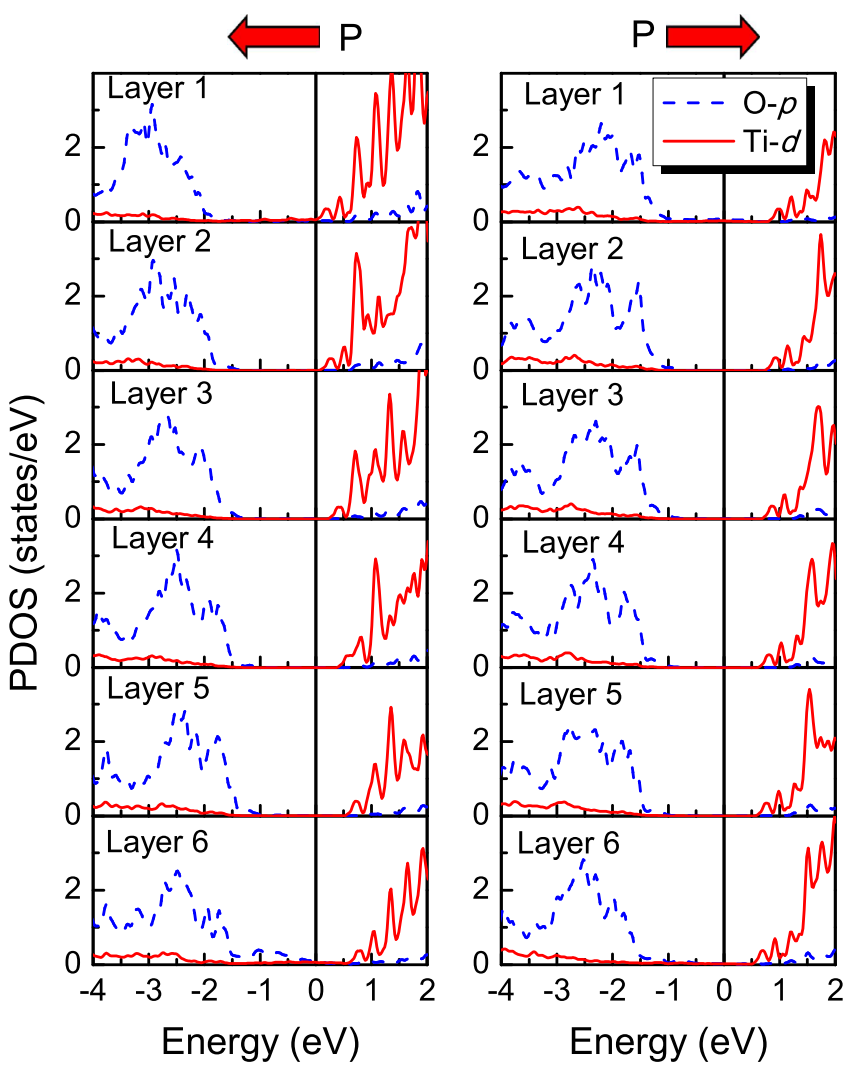

FIG. 8. Layer-resolved projected density of states (PDOS) for Ti-d (red solid) and O-p (blue dashed) orbitals for left (left panel) and right (right panel) polarization states as denoted by the arrows. The layer numbers are marked in Fig. 7(b). The Fermi level has been aligned to zero.

the FTJs was not taken into account in our calculations. We have calculated the structural parameters for one-unitcell PTO by including the SOC and found that there is no significant difference between the structural parameters calculated with SOC and that without SOC. Thus the ferroelectricity is expected to be affected not much by SOC. Regarding to the transport calculations for the obtention of TER, we also neglected the SOC. Besides the realistic first-principles calculations by including the SOC are computationally very demanding. Regarding to the role of PTO barrier, the asymmetry of PTO barrier plays a key role for the TER effects, which will not be affected much by the SOC in PTO as discussed above. Regarding to the role of electrodes, actually the electrodes provide the electron transport channels that go through the junction and the bands of electrodes will be spin-split induced by the SOC but the number of channels is not changed. Thus it is expected that the conductance of FTJ will also not be affected much by the SOC in electrodes. In a sense, we may say, that the SOC does not enter the TER physics and it will change our results quantitatively but not qualitatively. This is possibly the reason why most of previous work neglects the SOC in transport calculations even with heavy-element electrodes. ${ }^{23,35,36}$ Even so, the effect of SOC on the ferroelectric and TER for the FTJs should be further explored by first-principles calculations by including the SOC and we hope to clarify these issues in a future study. 


\section{CONCLUSIONS}

In conclusion, we have studied the ferroelectricity and TER effect in PTO-based FTJs by means of first-principles calculations. Our calculations suggest that the ferroelectricity for s-FTJs depend crucially on both metal electrode type and PTO interface termination, which is due to different screening lengths of metal electrodes. Moreover, we find significant asymmetric displacements in the a-FTJs resulting from intrinsic built-in electric field due to the work functions difference between metal electrodes. Interestingly, through quantum transport calculations on two prototypical Pt/PTO/ Fe and SRO/PTO/Pt a-FTJs, giant TER effects are predicted. The physical origin of TER effect is clarified through the real-space scattering state and complex band structure. Our results provide some fundamental understandings of ferroelectricity and TER mechanism in FTJs and give some practical guidelines for the designing of FTJ-based devices.

\section{ACKNOWLEDGMENTS}

This work is supported by the University Grant Council of Hong Kong (Contract No. AoE/P-04/08). The atomic structure visualization is plotted by using VESTA software. ${ }^{47}$ We are grateful to the National Supercomputer Center in Tianjin for providing the computational facility.

${ }^{1}$ J. F. Scott, Science 315, 954 (2007).

${ }^{2}$ T. Tybell, C. H. Ahn, and J.-M. Triscone, Appl. Phys. Lett. 75, 856 (1999).

${ }^{3}$ D. D. Fong, G. Brian Stephenson, S. K. Streiffer, J. A. Eastman, O. Auciello, P. H. Fuoss, and C. Thompson, Science 304, 1650 (2004).

${ }^{4}$ V. Garcia, S. Fusil, K. Bouzehouane, S. Enouz-Vedrenne, N. D. Mathur, A. Barthelemy, and M. Bibes, Nature 460, 81 (2009).

${ }^{5}$ E. Y. Tsymbal and H. Kohlstedt, Science 313, 181 (2006).

${ }^{6}$ V. Garcia and M. Bibes, Nat. Commun. 5, 4289 (2014).

${ }^{7}$ M. Ye. Zhuravlev, R. F. Sabirianov, S. S. Jaswal, and E. Y. Tsymbal, Phys. Rev. Lett. 94, 246802 (2005).

${ }^{8}$ A. Zenkevich, M. Minnekaev, Yu. Matveyev, Yu. Lebedinskii, K. Bulakh, A. Chouprik, A. Baturin, K. Maksimova, S. Thiess, and W. Drube, Appl. Phys. Lett. 102, 062907 (2013).

${ }^{9}$ R. Soni, A. Petraru, P. Meuffels, O. Vavra, M. Ziegler, S. Keun Kim, D. Seok Jeong, N. A. Pertsev, and H. Kohlstedt, Nat. Commun. 5, 5414 (2014).

${ }^{10}$ J. P. Velev, C.-G. Duan, J. D. Burton, A. Smogunov, M. K. Niranjan, E. Tosatti, S. S. Jaswal, and E. Y. Tsymbal, Nano Lett. 9, 427 (2009).

${ }^{11}$ M. Ye. Zhuravlev, Y. Wang, S. Maekawa, and E. Y. Tsymbal, Appl. Phys. Lett. 95, 052902 (2009).

${ }^{12}$ Y. W. Yin, J. D. Burton, Y.-M. Kim, A. Y. Borisevich, S. J. Pennycook, S. M. Yang, T. W. Noh, A. Gruverman, X. G. Li, E. Y. Tsymbal, and Q. Li, Nat. Mater. 12, 397 (2013).

${ }^{13}$ L. L. Tao and J. Wang, Appl. Phys. Lett. 108, 062903 (2016).

${ }^{14}$ Z. Wen, C. Li, D. Wu, A. Li, and N. Ming, Nat. Mater. 12, 617 (2013).

${ }^{15}$ A. Crassous, V. Garcia, K. Bouzehouane, S. Fusil, A. H. G. Vlooswijk, G. Rispens, B. Noheda, M. Bibes, and A. Barthelemy, Appl. Phys. Lett. 96, 042901 (2010).
${ }^{16}$ V. Garcia, M. Bibes, L. Bocher et al., Science 327, 1106 (2010).

${ }^{17}$ D. Pantel, S. Goetze, D. Hesse, and M. Alexe, Nat. Mater. 11, 289 (2012).

${ }^{18}$ J. G. Simmons, Phys. Rev. Lett. 10, 10 (1963).

${ }^{19}$ Y. Liu, X. Lou, M. Bibes, and B. Dkhil, Phys. Rev. B 88, 024106 (2013).

${ }^{20}$ Y. Umeno, J. Michael Albina, B. Meyer, and C. Elsässer, Phys. Rev. B 80, 205122 (2009).

${ }^{21}$ J. Lee, N. Sai, T. Cai, Q. Niu, and A. A. Demkov, Phys. Rev. B 81, 144425 (2010).

${ }^{22}$ M.-Q. Cai, Y. Zheng, P.-W. Ma, and C. H. Woo, J. Appl. Phys. 109, 024103 (2011).

${ }^{23}$ X. Luo, Y. Zheng, and B. Wang, J. Appl. Phys. 111, 074102 (2012).

${ }^{24}$ P. E. Blöchl, Phys. Rev. B 50, 17953 (1994); G. Kresse and D. Joubert, ibid. 59, 1758 (1999).

${ }^{25}$ G. Kresse and J. Furthmüller, Phys. Rev. B 54, 11169 (1996).

${ }^{26}$ J. P. Perdew and A. Zunger, Phys. Rev. B 23, 5048 (1981).

${ }^{27}$ T. Shimada, T. Ueda, J. Wang, and T. Kitamura, Phys. Rev. B 87, 174111 (2013).

${ }^{28}$ Y. Umeno, B. Meyer, C. Elsässer, and P. Gumbsch, Phys. Rev. B 74, 060101(R) (2006)

${ }^{29}$ J. Taylor, H. Guo, and J. Wang, Phys. Rev. B 63, 245407 (2001).

${ }^{30}$ W. A. Al-Saidi and A. M. Rappe, Phys. Rev. B 82, 155304 (2010).

${ }^{31}$ B. Meyer and D. Vanderbilt, Phys. Rev. B 65, 104111 (2002).

${ }^{32}$ M. Gajdoš, K. Hummer, G. Kresse, J. Furthmüller, and F. Bechstedt, Phys. Rev. B 73, 045112 (2006).

${ }^{33}$ R. R. Mehta, B. D. Silverman, and J. T. Jacobs, J. Appl. Phys. 44, 3379 (1973).

${ }^{34}$ G. Gerra, A. K. Tagantsev, N. Setter, and K. Parlinski, Phys. Rev. Lett. 96, 107603 (2006).

${ }^{35}$ J. P. Velev, C.-G. Duan, K. D. Belashchenko, S. S. Jaswal, and E. Y. Tsymbal, Phys. Rev. Lett. 98, 137201 (2007).

${ }^{36}$ L. Shen, T. Zhou, Z. Bai, M. Zeng, J. Q. Goh, Z. M. Yuan, G. Han, B. Liu, and Y. P. Feng, Phys. Rev. B 85, 064105 (2012).

${ }^{37}$ We calculated the WF using a slab separated by a vacuum of $\sim 20 \AA$. The WF is the minimum energy required to remove an electron from the Fermi level of a metal to a reference point sufficiently far from the metal surface, this reference point is often chosen as the vacuum level. And thus the work function can be obtained from the energy difference between the Fermi level of metal and the vacuum level, as shown in Fig. 4(a).

${ }^{38}$ W. J. Chen, Y. Zheng, X. Luo, B. Wang, and C. H. Woo, J. Appl. Phys. 114, 064105 (2013).

${ }^{39}$ A. Chanthbouala, A. Crassous, V. Garcia, K. Bouzehouane, S. Fusil, X. Moya, J. Allibe, B. Dlubak, J. Grollier, S. Xavier, C. Deranlot, A. Moshar, R. Proksch, N. D. Mathur, M. Bibes, and A. Barthelemy, Nat. Nanotechnol. 7, 101 (2012).

${ }^{40}$ X. Lu, H. Li, and W. Cao, J. Appl. Phys. 112, 054102 (2012).

${ }^{41}$ L. L. Tao, S. H. Liang, D. P. Liu, H. X. Wei, J. Wang, and X. F. Han, Appl. Phys. Lett. 104, 172406 (2014).

${ }^{42}$ O. Wunnicke, N. Papanikolaou, R. Zeller, P. H. Dederichs, V. Drchal, and J. Kudrnovský, Phys. Rev. B 65, 064425 (2002).

${ }^{43} \mathrm{Ph}$. Mavropoulos, N. Papanikolaou, and P. H. Dederichs, Phys. Rev. Lett. 85, 1088 (2000).

${ }^{44}$ L. L. Tao, S. H. Liang, D. P. Liu, and X. F. Han, J. Appl. Phys. 114, 213906 (2013).

${ }^{45}$ P. Giannozzi, S. Baroni, N. Bonini et al., J. Phys.: Condens. Matter 21, 395502 (2009); An energy cutoff of 40 Ry (400 Ry) for wavefunctions (charge density), $10 \times 10 \times 10 \mathrm{k}$-point mesh and $\mathrm{LDA}^{26}$ for the exchange and correlation functional are used in our calculations.

${ }^{46}$ M. Stengel, P. Aguado-Puente, N. A. Spaldin, and J. Junquera, Phys. Rev. B 83, 235112 (2011).

${ }^{47}$ K. Momma and F. Izumi, J. Appl. Crystallogr. 44, 1272 (2011). 